interpretive routine for the LGP-30, and (23), arccosh 250.001 (i.e. In 500) was found correct to 4 decimal places with $k=5$ (6 square roots) and to 11 places with $k=10$ (11 square roots).

The convergence of the sequence of approximations is only first order. At some cost in programming effort, it would be clearly possible to increase the convergence by one of the standard extrapolation techniques for accelerating the approach to the limit. However, the excellent convergence already present makes it unlikely that this device would be worthwhile unless the need for high accuracy was such that it was essential to keep $k$ as low as possible.

A special case of (13), with $x=-1$, has been known for a long time. This expansion, which has the limit $\pi$, can be obtained as one-half the perimeter of a $2^{k}$-gon inscribed in a circle of unit radius [2]. However, the general case, and the expansions obtainable by retaining the fourth-degree terms in the series for $\cos x$ and $\cosh x$ appear to be new.

Argonne National Laboratory

Argonne, Illinois

1. P. C. HAMmer, "Iterative procedures for taking roots based on square roots," $M T A C$, v. 9,1955, p. 68 .

2. R. Courant \& H. Robbins, What is Mathematics?, Oxford University Press, London, 1941, p. 124.

\title{
An Eigenvalue Problem Arising In Mass And Heat Transfer Studies
}

\section{By J. S. Dranoff}

1. Introduction. In a recent paper [1], S. Katz has considered the problem of catalytic chemical reactions occurring on the inside surface of a cylindrical tube. For the case of laminar flow of reactant through such a tube, he has shown how one may generate basic kinetic data for the reaction in question from easily made overall conversion measurements. The interested reader is referred to the original paper for the details of this analysis and its application.

In order to make use of Katz's analysis, one must have on hand the solution to the following Sturm-Liouville type eigenvalue problem:

$$
\begin{aligned}
& \frac{d}{d x}\left(x \frac{d \phi_{n}(x)}{d x}\right)+\lambda_{n} 4 x\left(1-x^{2}\right) \phi_{n}(x)=0, \quad 0 \leqq x \leqq 1 \\
& \phi_{n}(x) \text { regular at } x=0 \\
& \phi_{n}{ }^{\prime}(1)=0
\end{aligned}
$$

where the $\phi_{n}(x)$ are the eigensolutions and the $\lambda_{n}$ are the eigenvalues, with $n=$ $0,1,2, \cdots$. The first boundary condition leads, as in the case of Bessel's functions, to the condition $\phi_{n}{ }^{\prime}(0)=0$.

Received November 14, 1960; revised February 9, 1961. The author is now affiliated with the Department of Chemical Engineering, Northwestern University, Evanston, Illinois. 
In addition to $\phi_{n}(x)$ and $\lambda_{n}$, it is also necessary to have available the numerical values of the following integrals in order to complete the analysis.

$$
\begin{aligned}
a_{n} & =\frac{\int_{0}^{1} \frac{x^{2}}{2} \phi_{n}(x) 4 x\left(1-x^{2}\right) d x}{N_{n}} \\
b_{n} & =\frac{\int_{0}^{1} 2 x \phi_{n}(x) d x}{N_{n}} \\
N_{n} & =\int_{0}^{1}{\phi_{n}}^{2}(x) 4 x\left(1-x^{2}\right) d x .
\end{aligned}
$$

The reader may recognize that $a_{n}$ and $b_{n}$ are the coefficients in the expansion of the functions $x^{2} / 2$ and $2 x / 4 x\left(1-x^{2}\right)$, respectively, in a series of the eigenfunctions $\phi_{n}(x)$, with a weighting function of $4 x\left(1-x^{2}\right)$. Further, the integral $N_{n}$ may be regarded as a normalization factor.

It should be noted that problems similar to (1), but with alternate boundary conditions, have been studied by many investigators in the past. The earliest work, which dates back to 1883 , seems to be that of Graetz [2], who considered the case in which the second boundary condition of $(1)$ is $\phi_{n}(1)=1$. Most recently, Brown [3] recalculated the solutions to Graetz's problem by numerical techniques using a digital computer. However, no solutions pertinent to the present boundary conditions are available. Hence, the solution to the problem (1) and the determination of the integrals (2), (3), and (4) was the objective of this work. Since no complete analytic solution was possible, it was decided to solve the problem by numerical means using a Burroughs Datatron 205 digital computer. In addition, an effort was also made to obtain an asymptotic analytic representation of the solutions to the eigenvalue problem.

The normalization $\phi_{n}(0)=1.0$, for every $n$, was adopted for convenience in numerical calculations, rather than $N_{n}=1$, as chosen in [1]. Examination of the defining differential equation in (1) shows that any eigensolution may be multiplied by an arbitrary scale factor and still remain a solution. Thus, it is perfectly legitimate to make this normalization.

2. The Asymptotic Solution. An asymptotic solution to the problem (1) with the specification that $\phi_{n}(0)=1.0$ was attempted for large $n$. The method used was exactly that used by Sellars, Tribus and Klein [4] in dealing with the Graetz problem. Their procedure was followed except as required by the different boundary conditions of (1). The result was a three-piece analytic approximation to the eigenfunction $\phi_{n}(x)$ and an equation for estimating the eigenvalues $\lambda_{n}$.

The actual solutions obtained were as follows:

$$
\begin{array}{cr}
\phi_{n}(x)=J_{0}\left(2 x \sqrt{\lambda_{n}}\right) & \text { for } x \text { near } 0 \\
\phi_{n}(x)=\frac{1}{\sqrt{\pi} \lambda_{n}^{1 / 4}} \frac{\cos \left[x\left(1-x^{2}\right) !^{1 / 2} \sqrt{\lambda_{n}}+\sqrt{\lambda_{n}} \arcsin x-\pi / 4\right]}{x^{1 / 2}\left(1-x^{2}\right)^{1 / 4}} & \text { for } 0<x<1.0 \\
\phi_{n}(x)=(-1)^{n} \sqrt{\frac{2}{3}}(1-x)^{1 / 2} J_{-1 / 3}\left(\frac{2}{3}(1-x)^{3 / 2} \sqrt{8 \lambda_{n}}\right) & \text { for } x \text { near } 1.0
\end{array}
$$


where the eigenvalues may be approximated by the expression

$$
\lambda_{n}=4(n+1 / 3)^{2} \text {. }
$$

These equations are valuable for checking the results of subsequent numerical solutions. In particular, (8) is most useful in predicting the approximate location of the eigenvalues $\lambda_{n}$.

Although it is difficult to predict the accuracy of these asymptotic expressions, one may expect them to be quite good for $n \geqq 5$, judging from the results of Sellars, et al.

3. The Numerical Solution. The numerical solution of (1) was carried out by a trial-and-error procedure. A value of $\lambda_{n}$ was assumed, and the differential equation was integrated numerically from $x=0.0$ to $x=1.0$, using a standard Runge-Kutta method [5]. The initial conditions $\phi_{n}(0)=1.0$ and $\phi_{n}{ }^{\prime}(0)=0$ were assumed, as discussed earlier. If the resultant solution satisfied the criterion that $\phi_{n}{ }^{\prime}(1)=0.0$, it was deemed an eigensolution of the problem and the corresponding $\lambda_{n}$ a true eigenvalue. If this condition was not satisfied, a new value of $\lambda_{n}$ was chosen and the process repeated until successive values of $\lambda_{n}$ agreed within six (6) significant figures. (It should be noted that all computations were done in automatic floating point form using ten (10) decimal digit words, consisting of a two (2) digit characteristic exponent and an eight (8) digit mantissa.)

Successive estimates of $\lambda_{n}$ were made according to the following formula:

$$
\lambda_{n}{ }^{(i+1)}=\lambda_{n}{ }^{(i)}-\frac{\left[\phi_{n}{ }^{\prime}(1)\right]^{(i)}}{m^{(i)}}
$$

where the superscript indicates the " $i$ th" iteration and the value of $m$ is given by

$$
m^{(i)}=\frac{\left[\phi_{n}{ }^{\prime}(1)\right]^{(i)}-\left[\phi_{n}{ }^{\prime}(1)\right]^{(i-1)}}{\lambda_{n}{ }^{(i)}-\lambda_{n}{ }^{(i-1)}} .
$$

Equations (9) and (10) represent a simple linear approximation to the $\phi_{n}{ }^{\prime}(x)-\lambda_{n}$ relationship near $x=1.0$. Since this method requires the results of two previous iterations in order to estimate a new value of $\lambda_{n}$, it could not be applied until the third iteration. The second approximation to $\lambda_{n}$ was therefore found by arbitrarily increasing the starting value by 0.5 . By using the predictions of equation (8) to estimate $\lambda_{n}{ }^{(1)}$, it was normally possible to make the calculations converge within four or five iterations.

A fixed interval size was used in each integration. The final value was chosen such that a smaller interval would not produce any change within six (6) significant figures in the calculated eigenfunctions and eigenvalues. The number of intervals was varied in the calculation of successive eigenfunctions, but was always greater than $25 n$. This criterion was established in order to provide for the increasing complexity of the $\phi_{n}(x)$ within the interval $[0,1]$ with higher values of $n$.

When the eigenvalues and corresponding eigensolutions were obtained, the integrals (2), (3), and (4) were evaluated by numerical integration on the computer, using a standard Simpson's rule integration procedure.

4. Results. The numerical solution described above was carried out for $n$ from 1 to 20 . Table 1 presents the results of these calculations in terms of the eigenvalues, 
TABLE 1

Numerically Calculated Eigenvalues, Eigenfunctions, and Derivatives

\begin{tabular}{r|c|c|c|c}
\hline$n$ & $\lambda_{n}$ & $\Phi_{n}(1)$ & $\phi_{n^{\prime}(1)}$ & $\Delta x^{*}$ \\
\cline { 2 - 3 } 1 & 6.41990 & -0.492517 & $-1.0 \times 10^{-10}$ & 0.02 \\
2 & 20.9654 & +0.395509 & $-4.0 \times 10^{-9}$ & 0.0100 \\
3 & 43.5417 & -0.345874 & $+2.3 \times 10^{-7}$ & 0.0050 \\
4 & 74.1341 & +0.314047 & $-3.7 \times 10^{-7}$ & 0.0050 \\
5 & 112.737 & -0.291253 & $+1.5 \times 10^{-6}$ & 0.0050 \\
6 & 159.347 & +0.273810 & $-1.5 \times 10^{-6}$ & 0.0050 \\
7 & 213.963 & -0.259852 & $+3.5 \times 10^{-5}$ & 0.0025 \\
8 & 276.583 & +0.248329 & $-7.5 \times 10^{-5}$ & 0.0025 \\
9 & 347.206 & -0.238591 & $+4.5 \times 10^{-7}$ & 0.0025 \\
10 & 425.835 & +0.230189 & $-2.5 \times 10^{-4}$ & 0.0025 \\
11 & 512.462 & -0.222865 & $+5.0 \times 10^{-7}$ & 0.0025 \\
12 & 607.093 & +0.216373 & $-9.6 \times 10^{-8}$ & 0.0025 \\
13 & 709.725 & -0.210568 & $-7.6 \times 10^{-7}$ & 0.0020 \\
14 & 820.360 & +0.205334 & $+5.3 \times 10^{-7}$ & 0.0020 \\
15 & 938.997 & -0.200580 & $+1.2 \times 10^{-7}$ & 0.0020 \\
16 & 1065.63 & +0.196234 & $-5.5 \times 10^{-6}$ & 0.0020 \\
17 & 1200.27 & -0.192237 & $+2.1 \times 10^{-5}$ & 0.0020 \\
18 & 1342.92 & +0.188546 & $-3.2 \times 10^{-6}$ & 0.0020 \\
19 & 1493.56 & -0.185120 & $+1.5 \times 10^{-5}$ & 0.0020 \\
20 & 1652.20 & +0.181929 & $+1.8 \times 10^{-6}$ & 0.0020 \\
\hline
\end{tabular}

* The number of steps used in the numerical integrations is equal to $1.0 / \Delta x$.

TABLE 2

The Coefficient and Normalization Integrals

\begin{tabular}{r|c|c|c}
\hline$n$ & $a_{n}$ & ${ }^{2}$ & $N_{n}$ \\
\cline { 2 - 3 } 1 & -0.243568 & -1.02664 & +0.190138 \\
2 & +0.124300 & +1.06525 & +0.107731 \\
3 & -0.080695 & -1.08411 & +0.075286 \\
4 & +0.0585046 & +1.09553 & +0.0578066 \\
5 & -0.0452500 & -1.10329 & +0.0469413 \\
6 & +0.0365248 & +1.10895 & +0.0395161 \\
7 & -0.0303940 & -1.11331 & +0.0341194 \\
8 & +0.0258748 & +1.11677 & +0.0300203 \\
9 & -0.0224156 & -1.11957 & +0.0268008 \\
10 & +0.0197100 & +1.12198 & +0.0242052 \\
11 & -0.0175137 & -1.12392 & +0.0220681 \\
12 & +0.0157222 & +1.12564 & +0.0202777 \\
13 & -0.0142302 & -1.12714 & +0.0187560 \\
14 & +0.0129707 & +1.12847 & +0.0174469 \\
15 & -0.0118950 & -1.12964 & +0.016309 \\
16 & +0.0109669 & +1.13069 & +0.0153098 \\
17 & -0.0101598 & -1.13165 & +0.0144264 \\
18 & +0.00945062 & +1.13250 & +0.0136393 \\
19 & -0.00882477 & -1.13229 & +0.0129337 \\
20 & +0.00826766 & +1.13400 & +0.0122975 \\
\hline
\end{tabular}


and the eigenfunctions and their derivatives evaluated at $x=1.0$. In addition, the size of the integration interval used in the numerical calculations has also been tabulated for reference. Note that the leading eigensolution for $n=0$, namely $\lambda_{0}=0, \quad \phi_{0}(x) \equiv 1$, has not been tabulated.

More extensive values of the eigenfunctions are shown in Table 4 for intervals of $x=0.1$. Complete tables are available through the Engineering Research Section of the American Cyanamid Company, Stamford, Connecticut.

The integrals (2), (3), and (4) were evaluated for each of the above functions and are presented in Table 2. The data of Tables 1 and 2 may now be used to evaluate the function

$$
M(\theta)=1+\sum_{n=1}^{\infty}\left(\lambda_{n} a_{n}+b_{n}\right) \phi_{n}(1) e^{-\lambda_{n} \theta}
$$

which is equation (48) of [1].

The values of $a_{0}, b_{0}$, and $N_{0}$ have not been tabulated. These may be readily seen to equal $\frac{1}{6}, 1$, and 1 , respectively, by examination of equations (2), (3), and (4).

Some idea of the validity of the asymptotic expansion described above can be obtained by comparing the calculated and predicted values of the eigenvalues as determined by numerical integration and equation (8), respectively. These data are shown in Table 3. It is apparent that the asymptotic expression for the eigenvalues is quite a good approximation for $n \geqq 5$. It is assumed that this agreement holds for the asymptotic forms of the eigensolution as well, although no specific test has been made of this point as yet.

TABLE 3

Test of the Asymptotic Expression for the Eigenvalues

\begin{tabular}{r|c|c|c}
\hline$n$ & $\lambda$ (Calculated) & $\lambda$ (Predicted by Equation 8$)$ & $\lambda_{\text {pred }} / \lambda_{\text {cal }}$ \\
\cline { 2 - 3 } 1 & 6.41990 & 7.11111 & 0.9028 \\
2 & 20.9654 & 21.7778 & 0.9627 \\
3 & 43.5417 & 44.4444 & 0.9797 \\
4 & 74.1341 & 75.1111 & 0.9870 \\
5 & 112.737 & 113.7778 & 0.9909 \\
6 & 159.347 & 160.444 & 0.9932 \\
7 & 213.963 & 215.111 & 0.9947 \\
8 & 276.583 & 277.778 & 0.9957 \\
9 & 347.206 & 348.444 & 0.9964 \\
10 & 425.835 & 427.111 & 0.9970 \\
11 & 512.462 & 513.778 & 0.9974 \\
12 & 607.093 & 608.444 & 0.9978 \\
13 & 709.725 & 711.111 & 0.9981 \\
14 & 820.360 & 821.778 & 0.9983 \\
15 & 938.997 & 940.444 & 0.9985 \\
16 & 1065.63 & 1067.11 & 0.9986 \\
17 & 1200.25 & 1201.78 & 0.9987 \\
18 & 1342.92 & 1344.44 & 0.9989 \\
19 & 1493.56 & 1495.11 & 0.9990 \\
20 & 1652.20 & 1653.78 & 0.9990 \\
\hline
\end{tabular}




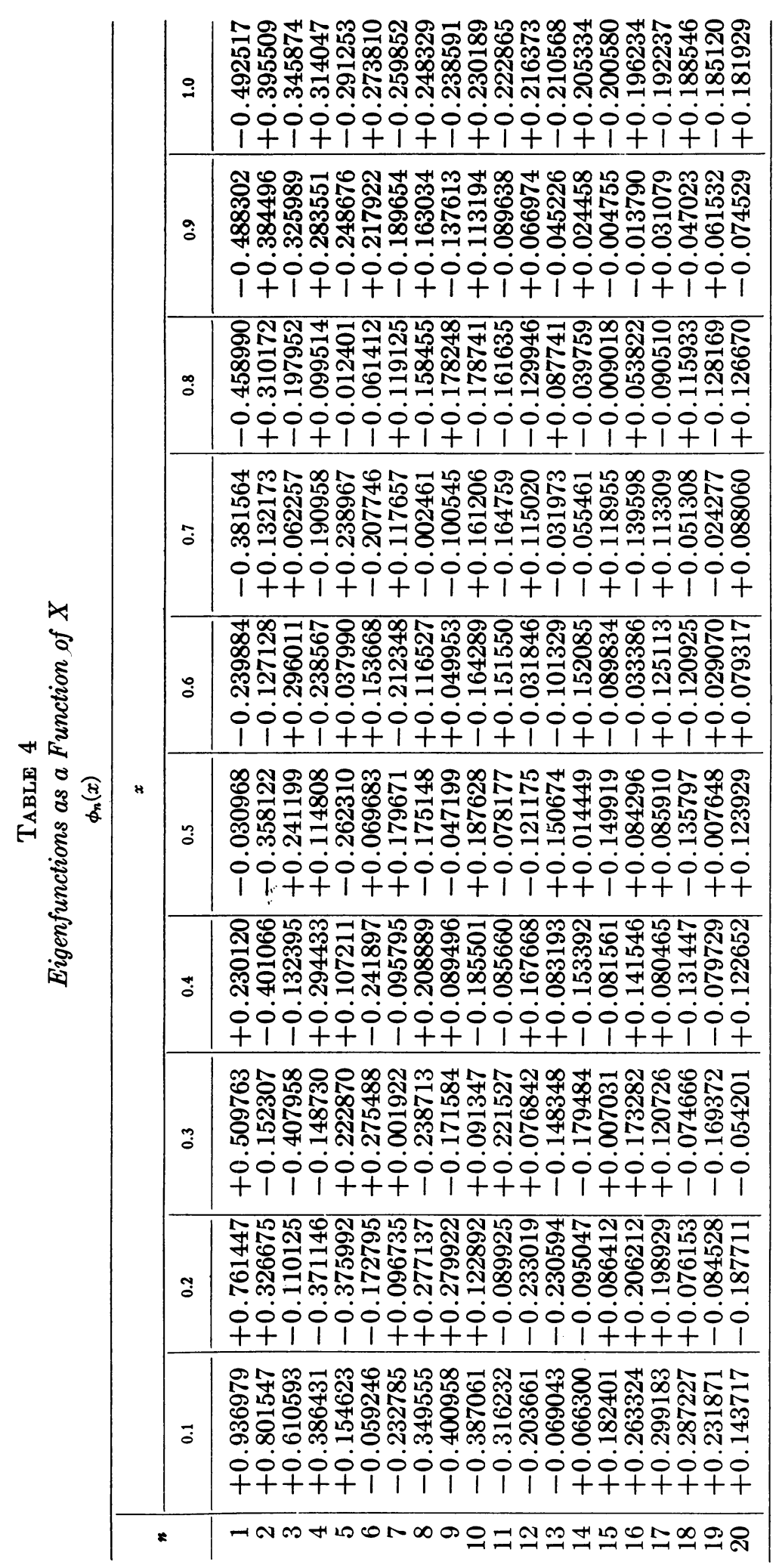


The recent work of Siegel, Sparrow and Hallman [6] has just come to the attention of the author. These workers have considered this problem in the heat transfer context. They report values of the eigenfunctions and eigenvalues which are in excellent agreement with the more extensive data of the present work.

5. Acknowledgment. The work reported here was carried out under the auspices of the Engineering Research Section of the American Cyanamid Company, Stamford, Connecticut. Permission to publish these results is hereby acknowledged with thanks.

The author is also indebted to S. Katz and J. Longfield of American Cyanamid for many helpful discussions concerning this problem.

Central Research Division

American Cyanamid Company

Stamford, Connecticut p. 202.

1. S. KAtz, "Chemical reactions catalyzed on a tube wall," Chem. Eng. Sci. v. 10, 1959,

2. L. Graetz, "Uber die Warmeleitungs fahigkeit von Flussigkeiten," Ann. Physik, v. 18,1883 , p. 79.

3. G. M. Brown, "Heat or mass transfer in a fluid in laminar flow in a circular or flat conduit," A. I. Ch. E. J., v. 6, 1960, p. 179.

4. J. B. Sellars, M. Tribus, \& J. S. Klein, "Heat transfer to laminar flow in a round tube or flat conduit- the Graetz problem extended," Trans. A. S. M.E., v. 78, 1956, p. 441.

5. F. B. Hildebrand, Introduction to Numerical Analysis, McGraw-Hill Publishing Co., New York, 1956, p. 238 .

6. R. Siegel, E. M. Sparrow \& T. M. Hallman, "Steady laminar heat transfer in a circular tube with prescribed wall heat flux," Appl. Sci. Res., v. A7, 1958, p. 386.

\section{Efficient Continued Fraction Approximations To Elementary Functions}

\section{By Kurt Spielberg}

1. Introduction. This paper describes an application and extension of the work of H. J. Maehly [1] on the rational approximation of $\operatorname{arc} \tan x$, and of E. G. Kogbetliantz [2], who developed Maehly's procedure so as to be applicable to the computer programming of elementary transcendental functions.

It is to be shown here that certain modifications, such as the introduction of terms which are easily computed on specific computers, lead to considerable improvements. In particular, the application of the modified method to several elementary functions will be described and corresponding final results will be given. Some of these approximations have been used with great success to develop subroutines for the IBM 704 and 709 computers. Our experience indicates that the method of Maehly and Kogbetliantz, as modified below, is superior to other current numerical procedures.

2. The Modified Method of Maehly and Kogbetliantz. The basic idea made use of by H. J. Maehly in connection with $f(x)=\operatorname{arc} \tan x$ is to approximate the function $f(x)$ by a ratio of two Chebyshev sums of order $k$

Received in final revised form December 21, 1960. 